\title{
Publicación científica y características de las tesis de salud de universidades del departamento de Puno, Perú
}

Oscar Javier Mamani-Benito* 1; Glubert Ramos Vilca ${ }^{2}$; Dawer Joe Rodrigo Ccaso 3; Fernando Quispe Mayta 4; Neyda Silene Chipana Paye ${ }^{3}$

\section{RESUMEN}

Con el objetivo de determinar la frecuencia de publicación de tesis de pregrado en salud e identificar sus características, se realizó un estudio transversal observacional en un conjunto de 482 tesis aprobadas entre 2016 y 2018 en carreras de salud de cuatro universidades del departamento de Puno. Las tesis correspondían a medicina $(25,10 \%)$, enfermería $(46,50 \%)$, nutrición $(18,5 \%)$ y psicología $(10,00 \%)$. Los resultados muestran que la mayor proporción de tesis fueron descriptivos $(56,80 \%)$ y de diseño no experimental $(90,50 \%)$; el 36,10\% involucró a la población hospitalaria y el $58,70 \%$ utilizó una muestra de entre 51 a 200 participantes. Además, solo cinco tesis $(1,00 \%)$ se publicaron en alguna revista científica indexada. Se concluye que la frecuencia de publicación de tesis de pregrado en carreras de salud de las universidades de Puno, es muy baja, además, que las investigaciones tienen un diseño básico de poco alcance y que la mayoría de las muestras son pequeñas.

Palabras clave: Publicaciones electrónicas; Educación de pregrado en medicina; Tesis académicas (Fuente: DeCS BIREME).

\section{Scientific publications and characteristics of health theses from universities of the department of Puno, Peru}

\section{ABSTRACT}

An observational cross-sectional study was conducted in order to determine the publication frequency of undergraduate health theses and identify their characteristics. The study population consisted of 482 health theses from four universities of the department of Puno approved between 2016 and 2018. Such theses belonged to the areas of medicine (25.10\%), nursing $(46.5 \%)$, nutrition (18.5\%) and psychology $(10.00 \%)$. The results show that $56.8 \%$ of the theses used a descriptive design, $90.5 \%$ used a non-experimental design, $36.1 \%$ involved the hospital population and $58.70 \%$ used a sample of 51 to 200 participants. Furthermore, only five theses $(1.00 \%)$ were published in an indexed scientific journal. It is concluded that there is a very low publication frequency of undergraduate health theses from universities of Puno, research studies use a basic design with a narrow scope and most research studies have a small sample size.

Keywords: Electronic publications; Education, medical, undergraduate; Academic dissertation (Source: MeSH NLM).

\footnotetext{
1 Universidad Peruana Unión. Juliaca, Puno, Perú.

2 Universidad Andina Néstor Cáceres Velásquez, Asociación Científica de Estudiantes de Medicina. Juliaca, Puno, Perú.

3 Universidad Peruana Unión, Escuela Profesional de Psicología. Juliaca, Puno, Perú.

4 Universidad Nacional del Altiplano, Sociedad Científica de Estudiantes de Medicina. Puno, Perú.

* Autor corresponsal
} 


\section{INTRODUCCIÓN}

Es necesario reconocer que en el Perú aún no se ha logrado instaurar una cultura de investigación entre los profesionales de salud. Los escasos reportes acerca de producción científica así lo demuestran, sobre todo, lo relacionado a publicación de artículos en revistas de alto impacto ${ }^{(1-3)}$.

En tal sentido, existen medios que podrían impulsar la generación de nuevo conocimiento en el ámbito de la salud. Uno de ellos es la tesis de pregrado que se desarrolla en las escuelas profesionales de medicina, enfermería, nutrición y psicología. Estas, además de ser un producto vienen a ser un medio formador para investigadores en el campo de la salud. Al respecto, la literatura científica peruana muestra algunos hallazgos de acuerdo a lo publicado en revistas científicas de la especialidad: la mayoría de estudios en el campo de la medicina revelan que existe un bajo porcentaje de publicación científica ${ }^{(4,5)}$.

Una situación similar se observa en contextos como la psicología, así lo demuestra el estudio de un conjunto de tesis en una universidad privada (con sede principal en Lima y filiales en Juliaca y Tarapoto) que halló que solo el $6 \%$ de 149 tesis lograron ser publicadas ${ }^{(6)}$.

Es por ello que la publicación de tesis debe generar un profundo interés y reflexión en las universidades peruanas. Se espera que todas las investigaciones culminen en la publicación científica, no obstante, no todas las tesis son publicables ${ }^{(7)}$. Por otro lado, en el contexto de la investigación formativa, la tesis es la primera oportunidad de hacer investigación ${ }^{(8)}$. Ante ello, el objetivo de este trabajo es determinar la frecuencia de publicación de tesis de pregrado en salud de universidades del departamento de Puno, e identificar sus características.

\section{EL ESTUDIO}

Se realizó un estudio descriptivo de análisis de documentos ${ }^{(9)}$ en un conjunto de 482 tesis aprobadas de carreras relacionadas a la salud (medicina, enfermería, psicología y nutrición) en el periodo de 2016 al 2018. El $64,50 \%$ de las tesis provenían de la Universidad Nacional del Altiplano (UNA); el 19,30 \% correspondían a la Universidad Andina Néstor Cáceres Velásquez (UANCV); el $15,1 \%$ se realizaron en la Universidad Peruana Unión (UPeU), y el $1 \%$, en la Universidad Privada San Carlos (UPSC). El 42,5\% se aprobaron en el año 2017; el 40,7 \%, en el 2018 y el 16,8 \%, en 2016.

Para recolectar los datos se revisó cada una de las tesis disponibles en el repositorio digital de cada institución, se registró la información respecto a características como universidad de procedencia, año de aprobación, tipo de estudio, tipo de población y tamaño muestral. Las variables como universidad de procedencia y año de aprobación se obtuvieron de la carátula principal de la tesis y las demás en el capítulo de la metodología; en algunos casos fue suficiente revisar el resumen. Por otra parte, para determinar la frecuencia de publicación se utilizó el buscador Google Académico y el motor de búsqueda de la base de datos Scopus. Para detectar las publicaciones se aplicó una combinación de tres términos de búsqueda (lugar de estudio, población de estudio y resultado principal), y también una búsqueda por autores con una estrategia empleada en otro estudio similar ${ }^{(10)}$ donde la ecuación usada para Google Académico consigna:

Autor: Luz Mercedes Mujica Calderón

«Luz M Mujica».

«Calderón MMM».

«Calderón LM».

«Mujica-Calderón L».

«Mujica-Calderón LM».

«Mujica L».

«Mujica $L M »$.

Mientras que la ecuación empleada para Scopus fue:

Autor: Luz Mercedes Mujica Calderón

Apellidos del autor: Mujica Calderón

Primer nombre del autor: Luz

Para la investigación no fue necesaria la aprobación de un comité de ética, pues las tesis registradas en el repositorio digital son de acceso libre. Luego de seleccionar las tesis según los criterios descritos, se digitaron en una base de datos en el programa Microsoft Excel (para Windows), se hizo una verificación de los datos y el control de calidad respectivo. Luego se obtuvo la estadística descriptiva del caso, para lo cual se usaron las frecuencias y porcentajes de cada variable medida.

En cuanto a los hallazgos, la tabla 1 muestra las características de las tesis de pregrado de facultades de ciencias de la salud de universidades de Puno en el periodo 2016 - 2018. Como se puede observar el $56,80 \%$ corresponde a estudios descriptivos, el $28,20 \%$ a correlacionales y en menor frecuencia los explicativos con un 3,70\%. En caso de los diseños, el 90,50\% son no experimentales, y $9,50 \%$ son pre o cuasiexperimenatles.

La población con mayor participación fue la hospitalaria (36,10\%), seguida de escolares $(23,90 \%)$, centros de salud $(14,50 \%)$, universitarios $(8,50 \%) \mathrm{y}$, en menor frecuencia, comunitaria $(5,40 \%)$ y penitenciaria $(1,20 \%)$. El análisis del tamaño muestral de los estudios revisados evidenció que el $58,70 \%$ tuvo de 51 a 200 participantes; el $27 \%$ involucró hasta 50; 12,4 \% contó entre 201 a 400; 1,2 \% tuvo más de 600 , y en $0,60 \%$ hubo una participación entre 401 y 600 personas. 
Publicación científica y características de las tesis de salud de universidades del departamento de Puno, Perú

Tabla 1. Características de las tesis de salud de universidades de Puno: periodo 2016 - 2018

\begin{tabular}{|c|c|c|c|}
\hline Variable & Categoría & Frecuencia & $\begin{array}{c}\text { Porcentaje } \\
(\%)\end{array}$ \\
\hline \multirow[t]{4}{*}{ Carrera profesional } & Enfermería & 224 & 46,50 \\
\hline & Medicina & 121 & 25,10 \\
\hline & Nutrición & 89 & 18,50 \\
\hline & Psicología & 48 & 10,00 \\
\hline \multirow[t]{3}{*}{ Año de aprobación } & 2016 & 81 & 16,80 \\
\hline & 2017 & 205 & 42,50 \\
\hline & 2018 & 196 & 40,70 \\
\hline \multirow[t]{4}{*}{ Universidad de procedencia } & UNA & 311 & 64,50 \\
\hline & UANCV & 93 & 19,30 \\
\hline & UPeU & 73 & 15,10 \\
\hline & UPSC & 5 & 1,00 \\
\hline \multirow[t]{7}{*}{ Tipo de población } & Escolar & 115 & 23,90 \\
\hline & Universitario & 41 & 8,50 \\
\hline & Centro de salud & 70 & 14,50 \\
\hline & Comunidad & 26 & 5,40 \\
\hline & Hospitalario & 174 & 36,10 \\
\hline & Penitenciario & 6 & 1,20 \\
\hline & Otro & 50 & 10,40 \\
\hline \multirow[t]{4}{*}{ Tipo de investigación } & Descriptivo & 274 & 56,80 \\
\hline & Correlacional & 136 & 28,20 \\
\hline & Explicativo & 18 & 3,70 \\
\hline & Otra & 54 & 11,20 \\
\hline \multirow[t]{3}{*}{ Diseño } & Experimental & 46 & 9,50 \\
\hline & No experimental & 436 & 90,50 \\
\hline & Hasta 50 participantes & 130 & 27,00 \\
\hline \multirow[t]{4}{*}{ Tamaño muestral } & Entre 51 a 200 & 283 & 58,70 \\
\hline & Entre 201 a 400 & 60 & 12,40 \\
\hline & Entre 401 a 600 & 3 & 0,60 \\
\hline & Mas de 600 & 6 & 1,20 \\
\hline
\end{tabular}

La tabla 2 muestra la frecuencia de publicación de tesis de salud de las universidades puneñas. Se observa que solo cinco trabajos de grado $(1,00 \%)$ lograron ser publicados en revistas científicas indexadas. De este grupo, solo se halló un artículo publicado en una revista indexada en la base de datos de SciELO, cuatro en revistas con indexaciones básicas y ninguna alcanzó a difundirse en alguna revista indexada en la base de datos Scopus. 
Tabla 2. Frecuencia de publicación científica de tesis de salud de universidades de Puno: periodo 2016 - 2018

\begin{tabular}{|c|c|c|c|}
\hline Variable & Categoría & Frecuencia & $\begin{array}{c}\text { Porcentaje } \\
(\%)\end{array}$ \\
\hline \multirow[t]{2}{*}{ Publicación científica } & Sí & 5 & 1,00 \\
\hline & No & 477 & 99,00 \\
\hline \multirow[t]{2}{*}{ Scopus } & Sí & 0 & 0,00 \\
\hline & No & 482 & 100,00 \\
\hline \multirow[t]{2}{*}{ SciELO } & Sí & 1 & 0,20 \\
\hline & No & 481 & 99,80 \\
\hline \multirow[t]{2}{*}{ Otros } & Sí & 4 & 0,80 \\
\hline & No & 478 & 99,20 \\
\hline
\end{tabular}

\section{DISCUSIÓN}

La presente investigación permite aproximarse a la realidad de la investigación formativa en salud de las universidades del departamento de Puno, Perú. El hecho de que solo cinco tesis culminaron con la etapa de la difusión de resultados en revistas científicas, corrobora en cierta medida lo mencionado por diversos investigadores respecto a la existencia de limitantes para la producción científica desde el pregrado ${ }^{(11-13)}$.

A diferencia de otras investigaciones, la frecuencia de publicación de tesis hallada en la presente investigación es muy baja (1,00\%). Por ejemplo, un estudio realizado en la Facultad de Medicina Humana de la Universidad San Martin de Porres mostró un 2,70 \% ${ }^{(10)}$; mientras que en la Facultad de Medicina Humana de la Universidad Nacional de San Antonio Abad del Cusco se halló un 5,00\% de un total de 398 tesis ${ }^{(4)}$; por otra parte, en otra investigación realizada en la Facultad de Medicina de la Universidad Pedro Ruiz Gallo se reportó un 4,10\% de 221 tesis ${ }^{(14)}$. Un porcentaje aún mayor se ha obtenido en una investigación realizada con trabajos para la obtención del grado de bachiller $(19,70 \%$ de 192) ${ }^{(15)}$. En la misma línea, un estudio realizado en la Universidad Nacional Mayor de San Marcos halló un 13,00 \% de 269 tesis ${ }^{(16)}$. Finalmente, un trabajo similar realizado en la Escuela de Psicología de la Universidad Peruana Unión, incluidas sus filiales en Juliaca y Tarapoto, encontró un $6,00 \%$ de $149^{(6)}$.

En cuanto a las características de los trabajos de grado, se halló una predominancia de tesis de tipo descriptivo, correlacional y de diseño no experimental. Al respecto, esto es similar a resultados de otras investigaciones, sobre todo en el ámbito de la medicina y psicología ${ }^{(6,14)}$. Esto significaria que, al iniciar el trabajo de grado, se intenta no complicar el proceso de la tesis, por lo que los tesistas realizan investigaciones sencillas que en muchos casos, son réplicas de investigaciones ya reportadas en la literatura académica. Aunado a esto, también influye el deseo de sustentar la tesis en el tiempo más breve ${ }^{(17)}$ y asi poder ejercer la profesión.

Con base en estos hallazgos, se asume que las tesis de pregrado de salud en las universidades de Puno son elaboradas en función de cumplir el requisito para obtener el título profesional y poder laborar en el campo profesional; esto deja de lado la oportunidad de aportar al conocimiento científico y la solución de problemas sanitarios de la región Puno y el país.

En conclusión, la frecuencia de publicación tesis de salud de universidades en el departamento de Puno es muy baja. Estas investigaciones se caracterizan, en mayor proporción, por emplear diseños no experimentales, de alcance descriptivo y con muestras relativamente pequeñas.

Contribuciones de los autores: Oscar Javier MamaniBenito concibió la idea y el diseño del estudio, realizó el análisis estadístico y la revisión crítica de la versión final del artículo. Glubert Ramos Vilca, Dawer Joe Rodrigo Ccaso, Fernando Quispe Mayta y Neyda Silene Chipana Paye recolectaron los datos y redactaron la primera versión del artículo.

Fuentes de financiamiento: Este artículo ha sido financiado por los autores.

Conflicto de interés: Los autores declaran no tener ningún conflicto de interés

\section{REFERENCIAS BIBLIOGRÁFICAS}

1. Luna-Solís Y. Producción científica en salud mental en Perú. Reto en 
tiempos de reforma de salud. Acta Méd Perú. 2015; 32(1): 36-40.

2. Taype-Rondán Á, Lajo-Aurazo Y, Huamaní C. Producción científica peruana sobre trastornos mentales en SciELO-Perú, 2006-2011. Rev Médica Hered. 2012; 23(3): 166.

3. Huaraca-Hilario CM, Apaza-Alcayhuaman A, Mejía CR. Publicación científica estudiantil en los últimos diez años: realidad peruana. Educ Méd Super. 2017; 31(3).

4. Atamari-Anahui N, Roque-Roque JS, Robles-Mendoza RA, Nina Moreno PI, Falcón-Huancahuiri BM. Publicación de tesis de pregrado en una facultad de Medicina en Cusco, Perú. Rev Médica Hered. 2015; 26(4): 217-21.

5. Taype-Rondán Á, Carbajal-Castro C, Arrunategui-Salas G, ChambiTorres J. Limitada publicación de tesis de pregrado en una facultad de medicina de Lima, Perú, 2000-2009. Ann Fac Med. 2012; 73(6): 153-7.

6. Mamani 0. Calidad metodológica y características de las tesis de pregrado de psicología de una universidad privada del Perú. Propos y Represent. 2018; 6(2): 301-19.

7. Miyahira J. Se necesita mejorar el proceso de elaboración de tesis en pregrado. Rev Médica Hered. 2018; 29(3): 135.

8. Mayta-Tristán P. Tesis en formato de artículo científico: oportunidad para incrementar la producción científica universitaria. Acta Méd Perú. 2016; 33(2): 95-8.

9. Montero I, León OG. A guide for naming research studies in psychology. Int J Clin Heal Psychol. 2007; 7(3): 847-62.

10. Mejía CR, Valladares-Garrido MJ, Almanza-Mío C, Benites-Gamboa D. Participación en una sociedad científica de estudiantes de medicina asociada a la producción científica extracurricular en Latinoamérica. Educ Médica. 2018; 20: 99-103.

11. Alarco J, Changllio-Calle G, Cahuana-Salazar M. Investigación en pregrado: interés según sexo y ciclo académico. Educ Médica. 2017; 18(1): 67-73.

12. Corrales-Reyes IE, Dorta-Contreras AJ. Students' scientific production: a proposal to encourage it. Medwave. 2018; 18(01): e7166.

13. Corrales-Reyes IE, Rodríguez M de J, Reyes JJ, García M. Limitantes de la producción científica estudiantil. Educ Médica. 2017; 18(3): 199-202.

14. Castro-Maldonado B, Callirgos-Lozada C, Caicedo-Písfil M, Plasencia-Dueñas E, Díaz-Vélez C. Características de las tesis de pre-grado de Medicina de una universidad pública del Perú. Horiz Med. 2015; 15(3): 34-9.

15. Osada J, Loyola-Sosa S, Ruiz-Grosso P. Publicación de trabajo de

conclusión de curso de estudiantes de medicina de una universidad peruana. Rev Bras Educ Médica. 2014; 38(3): 308-13.

16. Castro Rodríguez Y, Cósar-Quiroz J, Arredondo-Sierralta T, Sihuay-Torres K. Scientific production of theses supported and published by students of dentistry. Educ Médica. 2018; 19: 85-9.

17. Aiquipa J, Ramos C, Curay R, Guizado L. Factores implicados para realizar o no realizar tesis en estudiantes de psicología. Propos y Represent. 2018; 6(1): 21-52.

\section{Correspondencia:}

Javier Mamani-Benito

Dirección: Carretera Arequipa km 6 - Chullunquiani. Juliaca, Perú.

Teléfono: +51 959925651

Correo electrónico: oscar.mb@upeu.edu.pe

Recibido: 13 de enero de 2020

Evaluado: 04 de marzo de 2020 Aprobado: 27 de junio de 2020

(c) La revista. Publicado por Universidad de San Martín de Porres, Perú (c) $\mathbf{\text { BY }}$ Licencia de Creative Commons Artículo en acceso abierto bajo términos de Licencia Creative Commons Atribución 4.0 Internacional. (http://creativecommons.org/licenses/by/4.0/)

\section{ORCID iDs}

Oscar Mamani-Benito Glubert Ramos Vilca

Dawer Joe Rodrigo Ccaso Fernando Quispe Mayta Neyda Silene Chipana Paye
(1) https: / / orcid.org/0000-0002-9818-2601 (- https: / / orcid.org/0000-0003-3190-6748 (1) https: / / orcid.org/0000-0002-2657-4171 (- https: / / orcid.org/0000-0002-3601-2199 그 https: / / orcid.org/0000-0002-2119-8429 